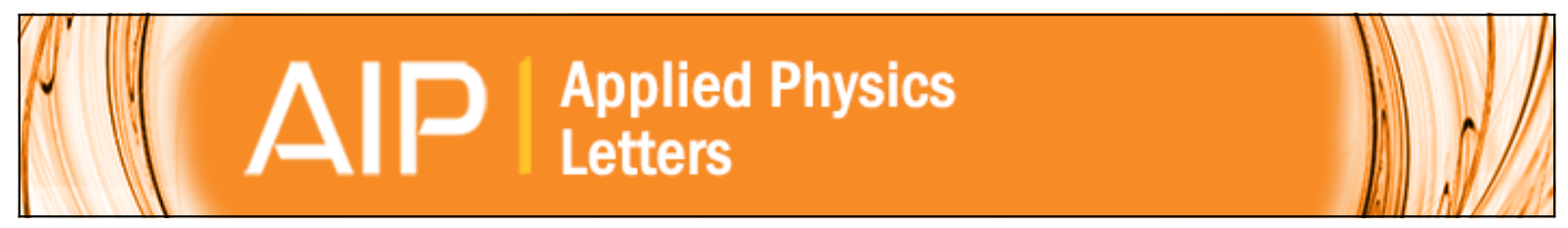

\title{
Remarkable enhancement of domain-wall velocity in magnetic nanostripes
}

Jun-Young Lee, Ki-Suk Lee, and Sang-Koog Kim

Citation: Applied Physics Letters 91, 122513 (2007); doi: 10.1063/1.2789176

View online: http://dx.doi.org/10.1063/1.2789176

View Table of Contents: http://scitation.aip.org/content/aip/journal/apl/91/12?ver=pdfcov

Published by the AIP Publishing

\section{Articles you may be interested in}

Mechanism of reversing the Neel domain walls in the Co nanostripes with transverse magnetic anisotropy Appl. Phys. Lett. 101, 252412 (2012); 10.1063/1.4772981

Phase diagram of magnetic domain walls in spin valve nano-stripes

Appl. Phys. Lett. 100, 172404 (2012); 10.1063/1.4704665

Magnetization reversal dynamics, nucleation, pinning, and domain wall propagation in perpendicularly magnetized ultrathin cobalt films: Influence of the Co deposition rate

J. Appl. Phys. 108, 093924 (2010); 10.1063/1.3506533

Periodic magnetic domain wall pinning in an ultrathin film with perpendicular anisotropy generated by the stray magnetic field of a ferromagnetic nanodot array

Appl. Phys. Lett. 94, 132504 (2009); 10.1063/1.3105988

Underlying mechanism of domain-wall motions in soft magnetic thin-film nanostripes beyond the velocitybreakdown regime

Appl. Phys. Lett. 93, 052503 (2008); 10.1063/1.2968138

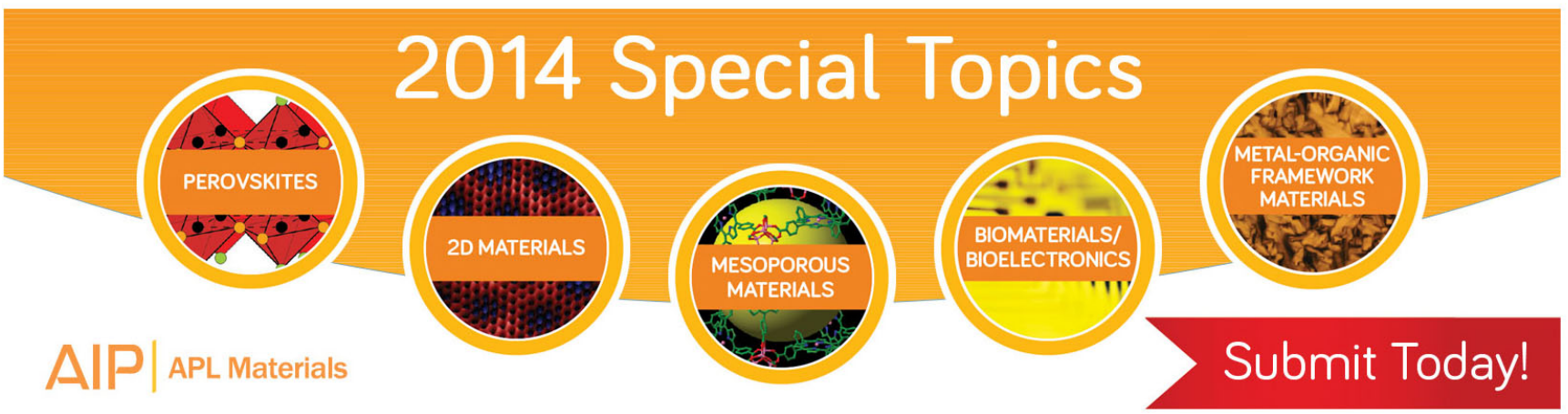




\title{
Remarkable enhancement of domain-wall velocity in magnetic nanostripes
}

\author{
Jun-Young Lee, Ki-Suk Lee, and Sang-Koog Kim ${ }^{\text {a) }}$ \\ Research Center for Spin Dynamics and Spin-Wave Devices and Nanospintronics Laboratory, Department \\ of Materials Science and Engineering, College of Engineering, Seoul National University, Seoul 151- \\ 744, Republic of Korea
}

(Received 11 April 2007; accepted 3 September 2007; published online 21 September 2007)

\begin{abstract}
Remarkable reductions in the velocity of magnetic-field (or electric current)-driven domain-wall (DW) motions in ferromagnetic nanostripes have typically been observed under magnetic fields stronger than the Walker threshold field [N. L. Schryer and L. R. Walker, J. Appl. Phys. 45, 5406 (1974)]. This velocity breakdown is known to be associated with an oscillatory dynamic transformation between transverse- and antivortex (or vortex)-type DWs during their propagations. The authors propose, as the result of numerical calculations, a simple means to suppress the velocity breakdown and rather enhance the DW velocities, using a magnetic underlayer of strong perpendicular magnetic anisotropy. This underlayer plays a crucial role in preventing the nucleation of antivortex (or vortex)-type DWs at the edges of nanostripes, in the process of periodic dynamic transformations from the transverse into antivortex- or vortex-type wall. The present study not only offers a promising means of the speedup of DW propagations to levels required for their technological application to ultrafast information-storage or logic devices, but also provides insight into its underlying mechanism. (C) 2007 American Institute of Physics. [DOI: 10.1063/1.2789176]
\end{abstract}

In recent years the high-velocity propagation of a single domain wall (DW) in ferromagnetic nanostripes driven by an applied magnetic field $H$ (Refs. 1-4) or spin-polarized current $I_{\text {sp }}$ (Refs. 5-9) has attracted considerable and growing interest, owing to its important applications to information-storage ${ }^{10,11}$ and logic ${ }^{12}$ devices. It is known that head-to-head (or tail-to-tail) transverse- and vortex-type DWs are stable in ferromagnetic nanostripes of submicron (or less) width under $H=0$, and that the DW type varies with the given width and thickness of the nanostripes. ${ }^{13}$ When a static field is applied to any type of DW in nanostripes, it propagates like a classical Newtonian particle in the $H$ direction to reduce the Zeeman energy. ${ }^{2-4,13}$ Intuitively, it is expected that the stronger the field the faster the DW motion, and hence the instant velocity $v$ of DWs increases linearly with increasing $H$. In real cases, however, the DW velocity remarkably drops above the Walker threshold field $H_{w}{ }^{1-4,13-16}$ This velocity breakdown is known to be associated with repeatable backward and forward motions of vortex walls (VWs) or antivortex walls (AVWs) that are periodically transformed, in their propagation above the $H_{w}$, from the transverse wall (TW) type into the AVW or VW type and back again. ${ }^{2,10}$ That velocity breakdown is undesirable in potential information-storage and logic devices based on the DW propagations because the DW velocity is an intrinsic factor determining the operating speed of such devices.

Thus, questions of how to increase the velocity of DW motions as well as to prevent velocity breakdown above the $H_{w}$ are currently key problems to be solved. It was suggested that some torques introduced from an orthorhombical anisotropy lead to an increase in the $H_{w}$ value and the maximum velocity, but this attempt could not exclude the periodic transformations of DWs above the $H_{w}{ }^{17,18}$ On the other

\footnotetext{
a) Author to whom correspondence should be addressed; electronic mail:
} sangkoog@snu.ac.kr hand, Nakatani et $a .^{2}$ recently proposed a counterintuitive way to suppress the velocity breakdown using the edge roughness ( $\sim 9 \mathrm{~nm}$ scale) of patterned nanostripes. However, the nanometer-scale edge roughness of nanostripes would not be effectively controllable in the fabrication process. In this letter, we propose an alternative way to suppress the DW velocity breakdown simply using a magnetic underlayer of strong perpendicular magnetic anisotropy (PMA). Moreover, we report the underlying mechanism of how this means effectively works.

In the present micromagnetic calculations, we used procedures similar to those in Ref. 19 and two different model systems: a rectangular cross-sectional permalloy $\left[\mathrm{Ni}_{80} \mathrm{Fe}_{20}\right.$ (Py) ] nanostripe with thickness of $11 \mathrm{~nm}$, width of $130 \mathrm{~nm}$, and length of $5 \mu \mathrm{m}$, and the same dimensional Py nanostripe, but with an FePt underlayer with thickness of $22 \mathrm{~nm}$, length of $5.5 \mu \mathrm{m}$, and width of $230 \mathrm{~nm}$, as displayed in Fig. 1. FePt was chosen because it is known to have a strong PMA. The material parameters for Py were as follows: the
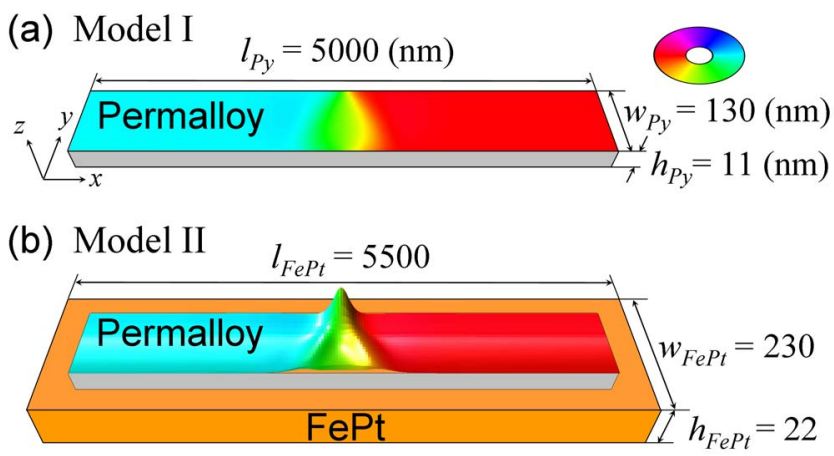

FIG. 1. (Color online) Model geometries and dimensions along with the equilibrium M configurations of a single DW of a TW type placed at the center in rectangular cross-sectional Py nanostripes (a) without (model I) and (b) with an FePt underlayer (model II). The in-plane and out-of-plane orientations of the local $\mathbf{M}_{s}$ in each Py nanostripe are noted by the color wheel and surface height, respectively. 


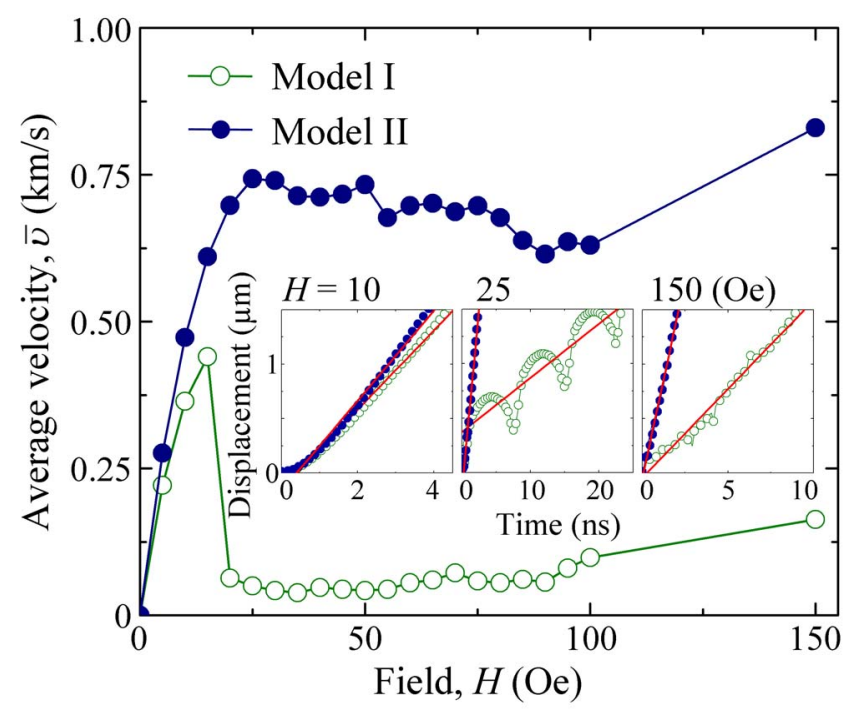

FIG. 2. (Color online) Average velocity of DW motions in the Py nanostripes vs $H$ for models I (open green circle) and II (closed blue circle). The representative $D$ vs $t$ curves for $H=10,25$, and 150 Oe are shown in the insets. The straight red lines are the results of fits to the data within the region up to $1.5 \mu \mathrm{m}$ from the initial DW position at $H=0$, indicating the corresponding $\bar{v}$ values at the indicated fields.

saturation magnetization, $M_{s}=8.6 \times 10^{5} \mathrm{~A} / \mathrm{m}$, and the exchange constant, $A_{\mathrm{ex}}=1.3 \times 10^{-11} \mathrm{~J} / \mathrm{m}$ with zero magnetocrystalline anisotropy, and for $\mathrm{FePt}, M_{s}=1.14 \times 10^{6} \mathrm{~A} / \mathrm{m}$, $A_{\mathrm{ex}}=1.54 \times 10^{-11} \mathrm{~J} / \mathrm{m}$, and the perpendicular magnetocrystalline anisotropy, $K_{\perp}=10^{7} \mathrm{~J} / \mathrm{m}^{3}$. The value of $A_{\mathrm{ex}}=1.4$ $\times 10^{-11} \mathrm{~J} / \mathrm{m}$ was used for the harmonized interlayer exchange constant between the different Py and FePt layers. The unit-cell size in the Py and FePt layers was $5 \times 5$ $\times 11 \mathrm{~nm}^{3}$ (Ref. 20) and the Gilbert damping constant was $\alpha=0.01$. To numerically calculate the dynamics of magnetization $\left(\mathbf{M}_{s}\right)$ as well as their interactions based on the LandauLifshitz-Gilbert equation of motion, ${ }^{21,22}$ we used the OOMMF (Ref. 23) code.

To carry out micromagnetic simulations on the dynamic motions of narrow DWs in nanostripes, first we used the equilibrium $\mathbf{M}$ configurations of a head-to-head TW-type $\mathrm{DW},{ }^{13}$ as shown in Fig. 1, which were obtained through the relaxation of an arbitrary TW-like configuration intentionally made at the center at $H=0$. Then, the motion of the TW was driven by an external $H$ applied in the $+x$ direction. The TW-type DWs start to move toward the field direction because the area of local $\mathbf{M}$, being parallel to the $H$ direction, grew larger through the DW motions, reducing the Zeeman energy. Such DW motions can be conceived as classicalparticle motions.

To examine contrasting DW motions for two different model nanostripes (models I vs II), the average velocity $\bar{v}$ of a single DW was plotted versus $H$ in Fig. 2. In the case of the Py nanostripe only (model I), the DW motion is relatively simple at small $H^{\prime}$ s $\left(<H_{w}\right)$. A linear increase of $\bar{v}$ is observed with increasing $H$ up to $H_{w} \sim 15 \mathrm{Oe}$, at which the peak value of $\bar{v}$ is $0.44 \mathrm{~km} / \mathrm{s}$. Above the $H_{w}$, the $\bar{v}$ value, however, decreases remarkably down to $0.04 \mathrm{~km} / \mathrm{s}$, as has been reported previously, ${ }^{14}$ so that the linear response of $\bar{v}$ with $H$ can be distinguished from an almost constant $\bar{v}$ response above $H_{w}$. The latter region is known as the oscillatory DW motion. Also, there is an additional region where $\bar{v}$ again increases with further increase of $H$ from $H \sim 100$ Oe. By contrast, in the same Py nanostripe on an FePt underlayer (model II), the $\bar{v}$ value is much increased up to $0.75 \mathrm{~km} / \mathrm{s}$ in the linear regime. Surprisingly, in the intermediate fields $\left(>H_{w}\right)$, the significant $\bar{v}$ reduction observed in model I is much suppressed, so that the $\bar{v}$ values are much greater, by one order of magnitude, than those in the Py nanostripe only. It seems that an underlayer of strong PMA surely plays a crucial role in the suppression of the velocity breakdown observed in the Py nanostripes without a PMA underlayer.

To better understand this remarkable suppression of the velocity breakdown, we show DW displacement-versus-time curves (hereafter denoted as $D$ vs $t$ curves) in the insets of Fig. 2, for the three representative cases, $H=10,25$, and 150 Oe. In both models, at $H=10 \mathrm{Oe}<H_{w}$, the DW moves steadily through the entire region of the nanostripes. However, at $H=25 \mathrm{Oe}>H_{w}$, the DW motions between the two models are remarkably contrasting. The oscillatory $D$ vs $t$ curve, representing an oscillatory DW motion observed in model I, turns into a linear curve, reflecting that the DW motion in model II was not oscillatory but steady. The periodic oscillation in the $D$ vs $t$ curve indicates that the DW moves forward in the initial propagation direction (the $H$ direction) and backward in the direction opposite to it in a periodic manner. $^{24}$ This surely leads to the remarkable reduction of $\bar{v}$. In fields $(H>100 \mathrm{Oe})$ much larger than $H_{w}$ the PMA underlayer also assists the acceleration of the DW motions in the Py nanostripe..$^{2-4,13}$ The $\mathbf{M}$ reversals in this field region occurs via the motion of multiple DWs, not by the motion of a single DW.

To elucidate the role of the FePt underlayer in the suppression of DW velocity breakdown, we examine the internal structures of the moving DWs along the nanostripes for the both models by showing snapshot images of the local $M_{x} / M_{s}$ components for each structure in the process of transformation from one type into another, where $\mathbf{M}_{x}$ is the $x$ component of M. For model I, the initially stable TW of $\Lambda$-shaped M configuration (noted as $\mathrm{TW}_{\Lambda}$ for its polarization) is transformed first into $A V W_{\text {down }}$, second to $T_{V}$, then to $A V W_{u p}$, and finally back to $\mathrm{TW}_{\Lambda}{ }^{24}$ The subscript in each DW type indicates the corresponding polarization $(\Lambda$ - or $\mathrm{V}$-shaped $\mathbf{M}$ configuration for TW and down or up core orientation for AVW). The AVW type switches between its down- and upcore orientations alternatively, as does the TW type in its periodic dynamic transformations. In contrast, for model II, the initially stable, single TW continues to move steadily in the Py nanostripe, without any transformation, as shown in the trajectories and the snapshot images of the $M_{x} / M_{s}$ components [Fig. 3(b)]. These results explain why the DW motion in model II is much faster than in model I.

Combined with the results shown in Fig. 3, the time variations of the total $E_{\text {tot }}$, exchange $E_{\text {ex }}$, dipolar $E_{\text {dip }}$, and Zeeman $E_{\text {Zeeman }}$ energy terms (Fig. 4) allow us to better understand the role of the underlayer in the suppression of velocity breakdown. In model I, the reduction of the velocity is related to the formation of the AVW type. Once the core of the AVW is well developed through its nucleation at a distance $(\sim$ the core size) far away from either edge, it continues to move inside the nanostripes due to its gyrotropic motion. $^{24}$ During this gyromotion, $E_{\mathrm{ex}}$ does not much change, but $E_{\text {Zeeman }}$ and $E_{\text {dip }}$ change according to the core position. As a consequence, $E_{\text {Zeeman }}$ for the AVW type first decreases and then increases because the AVW, due to its gyrotropic motion, moves first forward and then backward, 


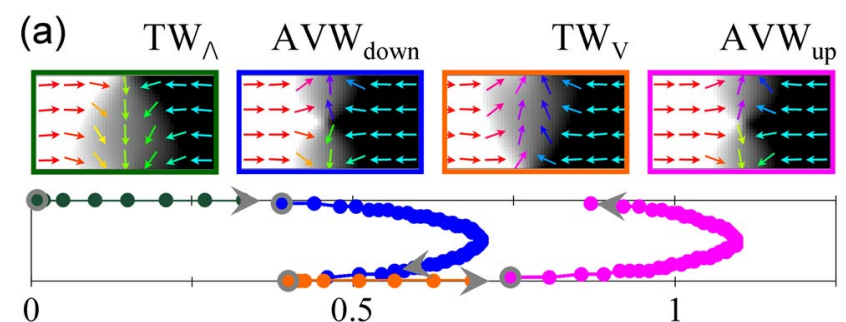

Distance along the long axis $(\mu \mathrm{m})$

(b)

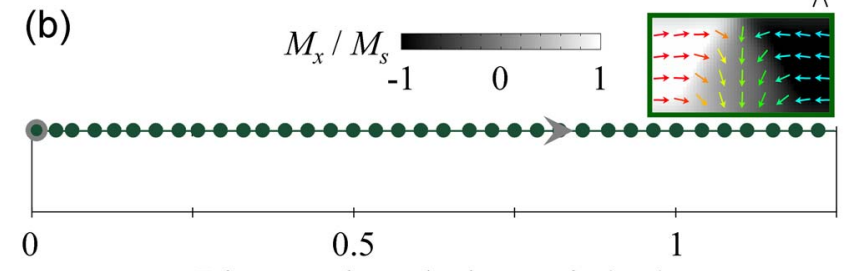

Distance along the long axis $(\mu \mathrm{m})$

FIG. 3. (Color online) Snapshot images of the dynamic evolution of the motion of a single DW. The top view images indicate the local values of the $M_{x} / M_{s}$ components for the dynamic structures of the individual DWs within the nanostripes (a) without and (b) with the FePt underlayer at $H=25$ Oe. The trajectories of the motions of the individual DW types were plotted according to the core position where the exchange energy was the maximum at each time. The arrows on the trajectory curves indicate the directions of the movement of each DW type. The open gray-colored circles represent the positions at which each DW type starts to move.

respectively, as evidenced by the trajectories shown in Fig. 3(a). When the TW type continues to move, $E_{\text {Zeeman }}$ decreases markedly due to its forward motion, whereas $E_{\mathrm{ex}}$ increases because the core of the TW type starts to move inside the nanostripe. By contrast, in model II, $E_{\text {dip }}$ and $E_{\text {ex }}$ do not oscillate in magnitude, but $E_{\text {Zeeman }}$ decreases significantly during the straightforward movement of the $\mathrm{TW}_{\Lambda}$ in
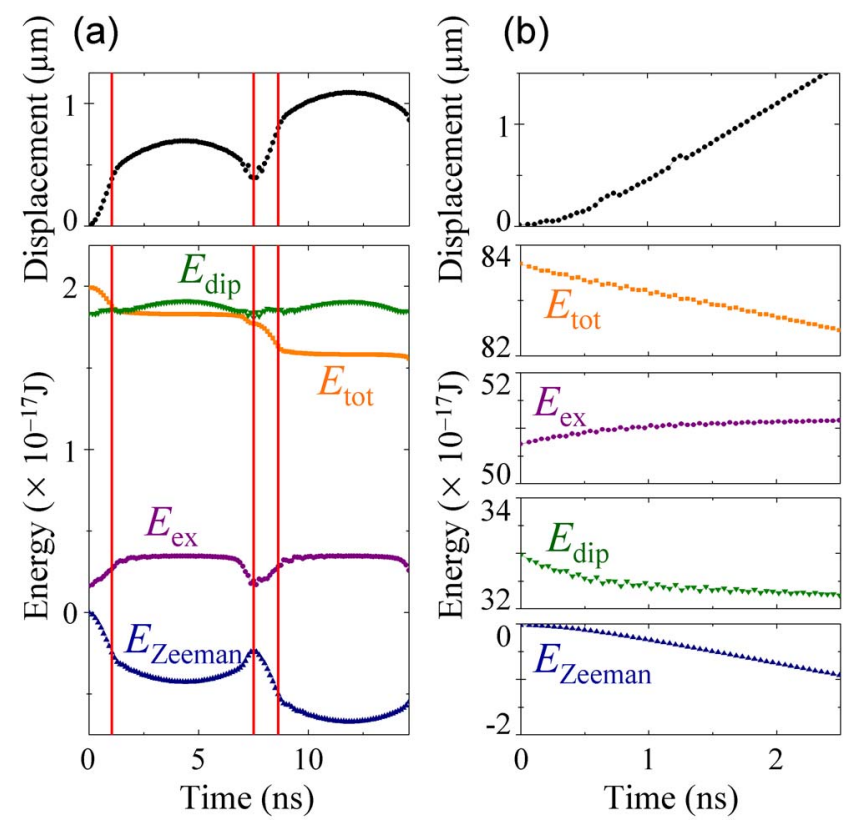

FIG. 4. (Color online) Energy variations with evolution time, along with the $D$ vs $t$ curves, for the Py nanostripes (a) without and (b) with the FePt underlayer. The colors indicate the individual energy terms as noted. The vertical red lines in (a) distinguish the individual regions of the motions of the different type DWs that transform from one type into another. the nanostripe, keeping its core at the top edge [Fig. 3(b)]. In the entire field region up to $150 \mathrm{Oe}$, a single TW propagates steadily along the nanostripe without any transformation into the AVW type, as evidenced by the internal structure of a moving single TW-type DW shown in Fig. 3(b). The underlayer of strong PMA introduces magnetostatic and exchange interactions between it and the Py nanostripe, which prevent the creation of the cores of AVWs near the edges of the nanostripe. Thus, the steady TW motion can be continued, yielding a much higher DW velocity than in the case without the FePt underlayer.

In conclusion, we found that the PMA underlayer plays an important role in preventing the nucleation of AVW (or VW) at the edges of nanostripes, which in turn hinders the gyrotropic motion of AVW (or VW) that would lead to oscillatory backward and forward motions. Thereby, the PMA underlayer significantly suppresses the typically observed velocity breakdown above the Walker critical field that results from the dynamic periodic change of the internal DW structure between the TW and AVW (or VW) types.

This work was supported by Creative Research Initiatives (ReC-SDSW) of MOST/KOSEF.

${ }^{1}$ R. Cowburn and D. Petit, Nat. Mater. 4, 721 (2005).

${ }^{2}$ Y. Nakatani, A. Thiaville, and J. Miltat, Nat. Mater. 2, 521 (2003).

${ }^{3}$ D. Atkinson, D. A. Allwood, G. Xiong, M. D. Cooke, C. C. Faulkner, and R. P. Cowburn, Nat. Mater. 2, 85 (2003).

${ }^{4}$ G. S. D. Beach, C. Nistor, C. Knutson, M. Tsoi, and J. L. Erskine, Nat. Mater. 4, 741 (2005).

${ }^{5}$ L. Thomas, C. Rettner, M. Hayashi, M. G. Samant, S. S. P. Parkin, A. Doran, and A. Scholl, Appl. Phys. Lett. 87, 262501 (2005).

${ }^{6}$ L. Thomas, M. Hayashi, X. Jiang, R. Moriya, C. Rettner, and S. S. P. Parkin, Nature (London) 443, 197 (2006).

${ }^{7}$ A. Yamaguchi, T. Ono, S. Nasu, K. Miyake, K. Mibu, and T. Shinjo, Phys. Rev. Lett. 92, 077205 (2004).

${ }^{8}$ M. Kläui, P.-O. Jubert, R. Allenspach, A. Bischof, J. A. C. Bland, G. Faini, U. Rüdiger, C. A. F. Vaz, L. Vila, and C. Vouille, Phys. Rev. Lett. 95, 026601 (2005)

${ }^{9}$ G. S. D. Beach, C. Knutson, C. Nistor, M. Tsoi, and J. L. Erskine, Phys. Rev. Lett. 97, 057203 (2006).

${ }^{10}$ M. Hayashi, L. Tomas, C. Rettner, R. Moriya, and S. S. P. Parkin, Nat. Phys. 3, 21 (2007).

${ }^{11}$ J. Grollier, P. Boulenc, V. Cros, A. Hamzić, A. Vaurès, A. Fert, and G. Faini, Appl. Phys. Lett. 83, 509 (2003).

${ }^{12}$ D. A. Allwood, G. Xiong, C. C. Faulkner, D. Atkinson, D. Petit, and R. P. Cowburn, Science 309, 1688 (2005).

${ }^{13}$ Y. Nakatani, A. Thiaville, and J. Miltat, J. Magn. Magn. Mater. 290-291, 750 (2005).

${ }^{14}$ N. L. Schryer and L. R. Walker, J. Appl. Phys. 45, 5406 (1974).

${ }^{15}$ S. W. Yuan and H. N. Bertram, Phys. Rev. B 44, 12395 (1991).

${ }^{16}$ B. N. Filippov, Low Temp. Phys. 28, 707 (2002).

${ }^{17}$ D. J. Breed, A. M. J. van der Heijden, H. Logmans, and A. B. Voermans, J. Appl. Phys. 49, 939 (1978).

${ }^{18}$ W. T. Stacy, A. B. Voermans, and H. Logmans, Appl. Phys. Lett. 29, 817 (1976).

${ }^{19}$ J.-Y. Lee, S. Choi, and S.-K. Kim, J. Magnetics 11, 74 (2006).

${ }^{20}$ To check whether the unit-cell size affects the simulation results, we additionally conducted the same simulations using a finer cell size of 2.5 $\times 2.5 \times 11 \mathrm{~nm}^{3}$, but the results of both cases agree quite well within less than $1 \%$ difference.

${ }^{21}$ S. Choi, K.-S. Lee, and S.-K. Kim, Appl. Phys. Lett. 89, 062501 (2006).

${ }^{22}$ K.-S. Lee, S. Choi, and S.-K. Kim, Appl. Phys. Lett. 87, 192502 (2005).

${ }^{23}$ M. J. Donahue and D. G. Porter, OOMMF code (see http://math.nist.gov/ oommf).

${ }^{24}$ J.-Y. Lee, K.-S. Lee, S. Choi, K. Y. Guslienko, and S.-K. Kim, Phys. Rev. $\mathrm{B}$ (in press). 